\title{
OBSERVATIONS OF STRAIGHT-ANGLE TAILED \\ RADIO GALAXIES IN RICH CLUSTERS OF GALAXIES
}

\author{
AILEEN A. O'DONOGHUE \\ St. Lawrence University, Canton, New York 13617 USA \\ JEAN A. EILEK \\ New Mexico Institute of Mining and Technology \\ Socorro, New Mexico 87801 USA \\ AND \\ FRAZER N. OWEN \\ NRAO-VLA, Socorro, New Mexico 87801 USA
}

\section{The SAT Multifrequency Study}

We have begun VLA observations of straight-angle tailed radio sources (SATs) at 1.5 and $4.8 \mathrm{GHz}$ (L and $\mathrm{C}$ band) to achieve one arcsecond resolution at each frequency. This will provide a SAT data set similar to the O'Donoghue, Owen, and Eilek (1990) WAT data set with both total intensity and spectral index information. We will use these data to examine morphological and dynamical properties of straight-tailed radio sources in clusters of galaxies.

The SATs in this study span a morphological range across the FRI/II classification. The current wisdom has it that radio sources are either FRtype I or type II and is supported by Owen's (1991) radio-optical diagram in which FRI and FRII sources occupy different parts of radio power/parent galaxy luminosity space. The SATs all fall into the FRI region of his plot, but some display FRII morphology. Are these the low radio power FRII's unrepresented in Eilek's (1996a) luminosity functions?

SATs have not been studied in a way comparable to NATs (eg. O'Dea and Owen 1987) or WATs (eg. O'Donoghue et al 1990, 1993, Loken et al 1995, Eilek 1996b, Katz-Stone et al 1996a), although a few individual SATs have been studied in great detail (eg. 3C31, Perley et al 1996; 3C449, KatzStone et al, 1996b). This is surprising because (1) they seem closest to the 
original picture of a type I source, without the complication of bending, and (2) inspection of the snapshot images from the Owen et al (1993) survey show that SATs comprise a greater range of morphology than NATs or WATs. We suspect they are, physically, the bridge between classic FRI and FRII sources.

\section{Selection of Sources}

The Owen et al (1993) cluster sample shows that sources which are type I's based on their radio and optical power, are more complex than was apparent in the $3 \mathrm{C}$ images upon which the FR classification was based. Approximately $1 / 3$ of the resolved sources in this sample fall into our SAT category and a variety of detail is found within this source type. The 8 sources we are observing span the morphological range, but are concentrated at the ends of the spectrum and at the transition point.

(1) Nearly FRII Sources: $0124+189$ and $0745+521$ Two sources that show well-collimated, bright jets with associated "backflow" lobes, although without the highly distinctive outer hot shot characteristic of the original FRII classification.

(2) Twin Tail Sources: 0306-237, 0738+441, and 1233+237 Three sources have less well-collimated jet flows and are reminiscent of the classic FRI sources such as $3 \mathrm{C} 449$. They also, however, show hints of broad "cocoons" surrounding the main jet, which suggests they may connect directly to the nearly FRII sources sources.

(3) Cocoon Sources: $0136+185,1530+282,2055-079$ Three sources show jets embedded in strong cocoons or halos, which extend as far as the end of the jets so that they could be either backflow or a lobe being shed as the source grows. The relative strengths of the jet and cocoon varies from source to source, making them appear transitional between the nearly FRII sources and the twin tail sources sources.

\section{References}

Eilek, J. A. (1996a), in preparation (luminosity functions).

Eilek, J. A. (1996b) submitted to Ap. J. (WATs).

Katz-Stone et al (1996a) in preparation (WATs).

Katz-Stone et al (1996b) in preparation (3C449).

Loken C. et al (1995) Ap. J. 445, 87.

O'Dea, C.P. \& Owen, F.N. (1987) Ap. J. 316, 95.

O'Donoghue, A.A., Owen, F.N. \& Eilek, J.A. (1990) Ap. J. Supp. 72, 75.

O'Donoghue, A.A., Eilek, J.A. \& Owen, F.N. (1993) Ap. J. 408, 428.

Owen, F. N. (1991), Steps Toward a Radio H-R Diagram, in Jets in Extragalactic Radio Sources, Proceedings of a Workshop Held at Ringberg Castle, Tegernsee, FRG, ed.

H.-J. Rser and K. Meisenheimer (Berlin:Springer-Verlag).

Owen, F.N. White, R.A. \& Ge, J. (1993) Ap. J. Supp. 87, 135.

Perley, R.A. et al (1996), in preparation. 